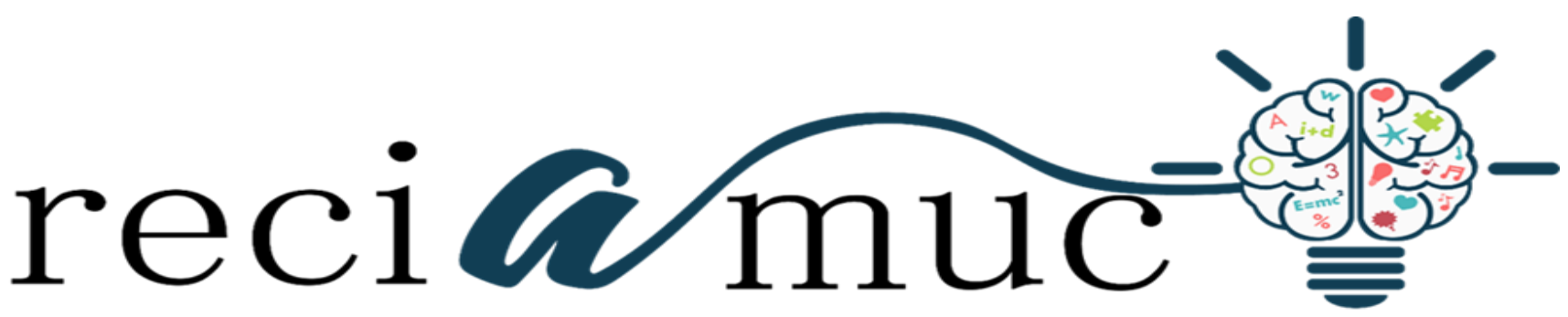

Revista cientifica de investigación actualización del mundo de las ciencias

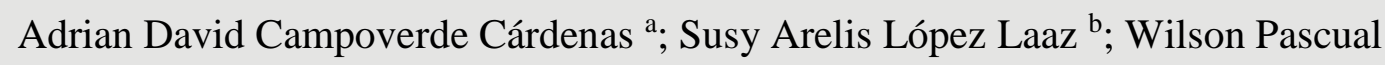

Correa Quinto ${ }^{\mathrm{c}}$; Jorge Daniel Cárdenas Rodríguez ${ }^{\mathrm{d}}$

Citometría de flujo en el diagnóstico de inmunopatía

Flow cytometry in the immunopathy diagnosis

Revista Científica de Investigación actualización del mundo de las Ciencias. Vol. 3 núm., 1, enero, ISSN: 2588-0748, 2018, pp. 218-241

DOI: $10.26820 /$ reciamuc/3.(1).enero.2019.218-241

URL: $\underline{\text { http://reciamuc.com/index.php/RECIAMUC/article/view/233 }}$

Código UNESCO: 3205 Medicina Interna

Tipo de Investigación: Artículo de Revisión

Editorial Saberes del Conocimiento

Recibido: 10/12/2018

Aceptado: 20/01/2019

Publicado: 30/01/2019

Correspondencia: director@reciamuc.com

a. Médico; Guayaquil, Ecuador.
b. Médico; Guayaquil, Ecuador.
c. Médico; Guayaquil, Ecuador.
d. Médico; Guayaquil, Ecuador. 


\section{Citometría de flujo en el diagnóstico de inmunopatía}

Vol. 3, núm. 1., (2019)

Adrian David Campoverde Cárdenas; Susy Arelis López Laaz; Wilson Pascual Correa Quinto; Jorge Daniel Cárdenas Rodríguez

\section{RESUMEN}

La citometría de flujo (CMF) es una técnica de avanzada, altamente sensible y automatizada, que se emplea para el inmunofenotipaje de las células normales y leucémicas. En este artículo se muestran los principales aspectos metodológicos a tener en cuenta para un mejor desarrollo e interpretación del inmunofenotipo por CMF, entre los que se encuentran: tipo, cantidad, conservación y transportación de la muestra, uso de anticoagulantes, empleo de anticuerpos monoclonales y fluorocromos, lisado de hematíes, fijación celular, así como la calibración y compensación de la auto-fluorescencia. Finalmente, se estudian las principales aplicaciones de esta metodología para definir el estado de maduración celular leucémico, clasificar las leucemias agudas en distintos subtipos inmunológicos, identificar subgrupos de mal pronóstico y detectar fenotipos aberrantes. El empleo de la citometría de flujo en el estudio de los Síndromes Linfoproliferativos crónicos (SLC) posee especial relevancia, al permitir un diagnóstico diferencial rápido entre una linfocitosis reactiva y un proceso monoclonal y en este caso contribuir a su filiación diagnóstico. El inmunofenotipo permite la clasificación diagnóstica de los SLC en neoplasias de origen B, T y NK. Además dentro de cada uno de estos subtipos de hemopatías se han podido definir subgrupos fenotípicamente diferentes con características clínico-biológicas específicas. El análisis de marcadores inmunológicos de leucemias y de Linfomsa no Hodgkin (LNH) permite una clasificación reproducible de esas malignidades. Así, la caracterización de estas malignidades se hace de acuerdo a criterios clínicos, morfológicos e inmunológicos que este tío de técnica permite analizar.

Palabras Claves: Citometría de Flujo; Inmunofenotipado; Leucemias Agudas; Inmunofenotipaje; Hematíes. 


\title{
Citometría de flujo en el diagnóstico de inmunopatía
}

Vol. 3, núm. 1., (2019)

Adrian David Campoverde Cárdenas; Susy Arelis López Laaz; Wilson Pascual Correa Quinto; Jorge Daniel Cárdenas Rodríguez

\begin{abstract}
Flow cytometry (CMF) is an advanced, highly sensitive and automated technique that is used for the immunophenotyping of normal and leukemic cells. This article shows the main methodological elements and is taken into account for a better development and interpretation of the immunophenotype by CMF, among which are: type, quantity, maintenance and transport of the sample, use of anticoagulants, use of monoclonal antibodies and Fluorochromes, red cell lysate, cell fixation, as well as calibration and compensation of auto-fluorescence. Finally, the main applications of this methodology to define the state of leukemic cell maturation, classify leukemias in different immunological subtypes, identify subgroups of poor prognosis and aberrant phenotypes. The use of flow cytometry in the study of chronic lymphoproliferative syndromes (SLC) has special relevance, as a single diagnosis, a reactive lymphocyte and a monoclonal process and in this case for its diagnostic filiation. The immunophenotype allows the diagnostic classification of SLC in neoplasms of B, T and NK origin. The specific characteristics of the clinical and biological characteristics. The analysis of immunological markers of leukemia and non-Hodgkin's lymphoma (NHL) allows a reproducible classification of these malignancies. Thus, the characterization of these malignant diseases is done according to the clinical, morphological and immunological criteria that this guy of the technique allows to analyze.
\end{abstract}

Key Words: Flow Cytometry; Immunophenotyping; Acute Leukemia; Immunophenotyping; Red Blood Cells. 


\section{Citometría de flujo en el diagnóstico de inmunopatía}

Vol. 3, núm. 1., (2019)

Adrian David Campoverde Cárdenas; Susy Arelis López Laaz; Wilson Pascual Correa Quinto; Jorge Daniel Cárdenas Rodríguez

\section{Introducción.}

La Citometría de Flujo (CMF) es una técnica de análisis celular multiparamétrico cuyo fundamento se basa en hacer pasar una suspensión de partículas (generalmente células) alineadas y de una en una por delante de un haz de láser focalizado. El impacto de cada célula con el rayo de luz produce señales que corresponden a diferentes parámetros de la célula y que son recogidos por distintos detectores. Estos convierten dichas señales en señales electrónicas que posteriormente serán digitalizadas para permitir la medida simultánea de varios parámetros en una misma célula.(Paredes, 2015).

En tal sentido, es importante entender que la técnica de la citometría de flujo identifica las células mediante el uso de anticuerpos monoclonales marcados con moléculas fluorescentes. Se utiliza para determinar el linaje, grado de maduración y presencia de fenotipos aberrantes en las células leucémicas, en el diagnóstico de los síndromes linfoproliferativos y de las leucemias agudas, en donde el análisis de la enfermedad mínima residual es de gran valor en el seguimiento de estas enfermedades tras el inicio del tratamiento, y en el diagnóstico de la hemoglobinuria paroxistica nocturna, entre otros.

La aplicación de esta técnica permite analizar la iluminación de las células con luz LASER, lo cual permite conocer el tamaño y la complejidad de los diferentes tipos de células. Ahora bien, si a estos resultados se le agrega el uso de moléculas fluorescentes para marcar estructuras celulares (por ej. ácido desoxirribonucleico o ADN, ácido ribonucleico o ARN, antígenos, etc.) se amplía enormemente sus posibilidades de detección. Dichas sustancias fluorescentes, al ser iluminadas por la luz LASER, emiten señales lumínicas en diferentes longitudes de onda (que 


\section{Citometría de flujo en el diagnóstico de inmunopatía}

Vol. 3, núm. 1., (2019)

Adrian David Campoverde Cárdenas; Susy Arelis López Laaz; Wilson Pascual Correa Quinto; Jorge Daniel Cárdenas Rodríguez

son captadas por diferentes detectores) con lo cual podemos obtener valiosa información de las propiedades de una población celular. (Paredes, 2015).

De allí, que en un citómetro convencional se logre obtener información sobre al menos 5 parámetros (por ejemplo tamaño, complejidad, contenido de ADN, viabilidad celular y presencia o ausencia de antígenos). Lo interesante de esta metodología es que no sólo aporta muchos datos en forma simultánea, sino que lo realiza a velocidades que parecen asombrosas porque es capaz de analizar al menos 1000 células en solamente un segundo.

Así también, los citómetros de flujo de mayor complejidad son también capaces de clasificar a las células que resulten de interés para el investigador. Entendemos por clasificar la posibilidad de separar del resto a un conjunto de células que comparten una o varias propiedades y recolectarlas en un tubo, placa de cultivo o portaobjeto. Esto permite llevar a cabo estudios más sofisticados de una población de células similares, iniciar cultivos de un mismo tipo celular, etc.

Por todo esto, para dar una idea de la especificidad del mecanismo de separación de los citómetros de flujo se debe mencionar que se emplean para separar los distintos tipos de cromosomas de la especie humana u otras especies. La obtención de suspensiones cromosómicas de elevada pureza mediante citometría de flujo ha permitido formidables avances en el conocimiento del genoma humano y en la obtención de sondas moleculares aplicables a diagnóstico citogenético de diversas patologías.

Por consiguiente, explicar en detalle el mecanismo que se utiliza para clasificar los diferentes tipos de células o cromosomas excede los objetivos de este artículo. Basta mencionar que para lograrlo se fragmenta la columna líquida en pequeñas gotitas haciendo vibrar la boquilla 


\section{Citometría de flujo en el diagnóstico de inmunopatía}

Vol. 3, núm. 1., (2019)

Adrian David Campoverde Cárdenas; Susy Arelis López Laaz; Wilson Pascual Correa Quinto; Jorge Daniel Cárdenas Rodríguez

a más de 20.000 veces por segundo. Luego de ajustes apropiados, se logra que cada gotita contenga una célula o cromosoma. Cuando el citómetro detecta una célula o cromosoma de interés para clasificar, el aparato carga eléctricamente la columna liquida de forma tal que sólo la gota que contiene el evento de interés (célula) queda cargada. Finalmente, el sistema direcciona dicha gota con la célula hacia un tubo recolector al ser desviada por un intenso campo eléctrico.

El campo de aplicación de este tipo de instrumentos crece rápidamente día a día ya que son numerosas las áreas de la biología y medicina que se benefician de su uso. Entre otras aplicaciones, se encuentra la tipificación de las leucemias y los linfomas que permite alcanzar un diagnóstico preciso, permitiendo un tratamiento y seguimiento más adecuado y eficaz. Otra aplicación muy importante es el estudio de las poblaciones linfocitarias afectadas por el virus de la inmunodeficiencia humana (VIH) causante del Síndrome de Inmunodeficiencia Adquirida (SIDA) que permite conocer en los individuos afectados su capacidad de respuesta inmunitaria, de capital importancia para el seguimiento de la evolución de la infección y el tratamiento adecuado para el paciente.

Por su parte, el estudio de la cantidad de material hereditario (ADN) posee numerosas aplicaciones. En medicina, se emplea para evaluar si existen cambios genéticos en poblaciones de células tumorales. La presencia de estos cambios tiene importancia en el prónostico de la enfermedad maligna. Dado el elevado número de células a analizar para realizar estos diagnósticos, el citómetro de flujo es la herramienta indicada para este tipo de estudios. También es posible estimar si las células han sufrido cambios relevantes en el ADN, así como evaluar su nivel de proliferación ya que con frecuencia las células malignas crecen con mayor rapidez que las normales. 


\section{Citometría de flujo en el diagnóstico de inmunopatía}

Vol. 3, núm. 1., (2019)

Adrian David Campoverde Cárdenas; Susy Arelis López Laaz; Wilson Pascual Correa Quinto; Jorge Daniel Cárdenas Rodríguez

En otras palabras, el citómetro es capaz de cuantificar el grado de proliferación celular, es decir la tasa con que las células se multiplican. En el campo de la biología, la citometría de flujo se ha tornado el método más empleado para determinar el contenido exacto de ADN de cada especie, lo cual es de suma importancia para estudios genéticos, evolutivos y biotecnológicos. A su vez, se pueden realizar estudios del nivel de ploidia, análisis cromosómicos y estudios del daño del ADN. En virtud de estos señalamientos, se establece la importancia del presente artículo, el cual centra su atención en analizar la "Cimetría de Flujo en el diagnóstico de Inmunopatía"

\section{Método.}

Para el desarrollo de este proceso investigativo, se plantea como metodología la encaminada hacia una orientación científica particular que se encuentra determinada por la necesidad de indagar en forma precisa y coherente una situación, en tal sentido, (Dávila R. , 2013), define la metodología "como aquellos pasos previos que son seleccionados por el investigador para lograr resultados favorables que le ayuden a plantear nuevas ideas”.(p.66)

Lo citado por el autor, lleva a entender que el desarrollo de la acción investigativa busca simplemente coordinar acciones enmarcadas en una revisión bibliográfica con el fin de complementar ideas previas relacionadas con la "Citometría de Flujo en el diagnóstico de Inmunopatía", para así finalmente elaborar un cuerpo de consideraciones generales que ayuden a ampliar el interés propuesto. 


\section{Citometría de flujo en el diagnóstico de inmunopatía}

Vol. 3, núm. 1., (2019)

Adrian David Campoverde Cárdenas; Susy Arelis López Laaz; Wilson Pascual Correa Quinto; Jorge Daniel Cárdenas Rodríguez

Tipo de Investigación

Dentro de toda práctica investigativa, se precisan acciones de carácter metodológico mediante las cuales, se logra conocer y proyectar los eventos posibles que la determinan, así como las características que hacen del acto científico un proceso interactivo ajustado a una realidad posible de ser interpretada. En este sentido, se puede decir, que la presente investigación corresponde al tipo documental, definido por (Castro, 2012), "se ocupa del estudio de problemas planteados a nivel teórico, la información requerida para abordarlos se encuentra básicamente en materiales impresos, audiovisuales y /o electrónicos". (p.41).

En consideración a esta definición, la orientación metodológicapermitió la oportunidad de cumplir con una serie de actividades inherentes a la revisión y lectura de diversos documentos donde se encontraron ideas explicitas relacionadas con los tópicos encargados de identificar a cada característica insertada en el estudio. Por lo tanto, se realizaron continuas interpretaciones con el claro propósito de revisar aquellas apreciaciones o investigaciones propuestas por diferentes investigadores relacionadas con el tema de interés, para luego dar la respectiva argumentación a los planteamientos, en función a las necesidades encontradas en la indagación.

\section{Fuentes Documentales}

El análisis correspondiente a las características que predomina en el tema seleccionado, llevan a incluir diferentes fuentes documentales encargadas de darle el respectivo apoyo y en ese sentido cumplir con la valoración de los hechos a fin de generar nuevos criterios que sirven de referencia a otros procesos investigativos. Para (Castro, 2012), las fuentes documentales incorporadas en la investigación documental o bibliográfica, "representa la suma de materiales 


\section{Citometría de flujo en el diagnóstico de inmunopatía}

Vol. 3, núm. 1., (2019)

Adrian David Campoverde Cárdenas; Susy Arelis López Laaz; Wilson Pascual Correa Quinto; Jorge Daniel Cárdenas Rodríguez

sistemáticos que son revisados en forma rigurosa y profunda para llegar a un análisis del fenómeno".(p.41). Por lo tanto, se procedió a cumplir con la realización de una lectura previa determinada por encontrar aquellos aspectos estrechamente vinculados con el tema, para luego explicar mediante un desarrollo las respectivas apreciaciones generales de importancia.

\section{Técnicas para la Recolección de la Información}

La conducción de la investigación para ser realizada en función a las particularidades que determinan a los estudios documentales, tiene como fin el desarrollo de un conjunto de acciones encargadas de llevar a la selección de técnicas estrechamente vinculadas con las características del estudio. En tal sentido, (Bolívar, 2015), refiere, que es "una técnica particular para aportar ayuda a los procedimientos de selección de las ideas primarias y secundarias”. (p. 71).

Por ello, se procedió a la utilización del subrayado, resúmenes, fichaje, como parte básica para la revisión y selección de los documentos que presentan el contenido teórico. Es decir, que mediante su aplicación de estas técnicas se pudo llegar a recoger informaciones en cuanto a la revisión bibliográfica de los diversos elementos encargados de orientar el proceso de investigación. Tal como lo expresa, (Bolívar L. , 2010)“las técnicas documentales proporcionan las herramientas esenciales y determinantes para responder a los objetivos formulados y llegar a resultados efectivos" (p. 58). Es decir, para responder con eficiencia a las necesidades investigativas, se introdujeron como técnica de recolección el método inductivo, que hizo posible llevar a cabo una valoración de los hechos de forma particular para llegar a la explicación desde una visión general. 


\section{Citometría de flujo en el diagnóstico de inmunopatía}

Vol. 3, núm. 1., (2019)

Adrian David Campoverde Cárdenas; Susy Arelis López Laaz; Wilson Pascual Correa Quinto; Jorge Daniel Cárdenas Rodríguez

Asimismo, se emplearon las técnicas de análisis de información para la realización de la investigación que fue ejecutada bajo la dinámica de aplicar diversos elementos encargados de determinar el camino a recorrer por el estudio, según, (Bolívar L. , 2010), las técnicas de procesamiento de datos en los estudios documentales "son las encargadas de ofrecer al investigador la visión o pasos que debe cumplir durante su ejercicio, cada una de ellas debe estar en correspondencia con el nivel a emplear" (p. 123). Esto indica, que para llevar a cabo el procesamiento de los datos obtenidos una vez aplicado las técnicas seleccionadas, tales como: fichas de resumen, textual, registros descriptivos entre otros, los mismos se deben ajustar al nivel que ha sido seleccionado

\section{Resultados.}

\section{Citometría de Flujo}

La citometría de flujo (CMF), es técnica utilizada para analizar y definir el perfil inmunofenotípico de las células neoplásicas, así como para establecer presencia de inmunofenotipos aberrantes. Se basa en el empleo de anticuerpos monoclonales específicos, dirigidos contra proteínas de membrana o citoplasmáticas, que llevan apareado un fluorocromo para su detección y visualización mediante un sistema informático apropiado.(Carbajo, 2015)

La citometría de flujo, representa una técnica médica que permite el análisis simultáneo de varios parámetros celulares. A través de la misma se interpreta la información del tamaño y granularidad celular, en combinación con la intensidad de expresión de moléculas de superficie e intracelulares, permitiendo identificar un inmunofenotipo que distingue células leucémicas de sus contrapartes normales(Mayani, 2015). En efecto, la citometría de flujo representa un proceso 


\section{Citometría de flujo en el diagnóstico de inmunopatía}

Vol. 3, núm. 1., (2019)

Adrian David Campoverde Cárdenas; Susy Arelis López Laaz; Wilson Pascual Correa Quinto; Jorge Daniel Cárdenas Rodríguez

en el que células u otras partículas biológicas incluídas en un flujo de líquido isotónico son empujadas a pasar, alineadas y de una en una, por delante de uno o varios detectores capaces de recoger y medir diferentes características físicas y/o químicas de esas células o partículas, a la vez que son iluminadas por un haz de luz, habitualmente un láser. (Colmenárez, 2014)

En términos generales, las características celulares analizadas mediante esta tecnología son el reflejo de dos grandes grupos de parámetros: por un lado, los derivados de la luz que al incidir sobre la célula o partícula es dispersada y que se relacionan entre otras características con el tamaño y la granularidad celulares y, por otra parte los que se asocian con la luz generada como consecuencia de la presencia en la célula de fluorocromos, bien de forma natural (auto fluorescencia) o unidos a ella artificialmente

Desde su aparición, la citometría de flujo ha sufrido un importante desarrollo debido en gran medida a que por su carácter multidisciplinario se ha beneficiado de los avances ocurridos en los últimos años en campos diversos como la informática, la producción de anticuerpos monoc1onales, la química de los fluo115 rocromos, los procedimientos de tinción citoquímica, la electrónica, la óptica y la tecnología láser. (Wilson K, 2013). Todo ello unido a su progresiva utilización en el análisis automático y separación de células ha abierto nuevas perspectivas en el campo del diagnóstico clínico y la investigación biomédica. Así, esta tecnología en un principio sofisticada y restringida a laboratorios de investigación básica ha pasado a estar presente en los últimos años en la rutina de muchos laboratorios clínicos.(Colmenárez, 2014) 


\section{Citometría de flujo en el diagnóstico de inmunopatía}

Vol. 3, núm. 1., (2019)

Adrian David Campoverde Cárdenas; Susy Arelis López Laaz; Wilson Pascual Correa Quinto; Jorge Daniel Cárdenas Rodríguez

La CMF proporciona información de manera rápida y sensible sobre diferentes características físicas y químicas de células en suspensión, siendo esta información de tipo cualitativo, cuantitativo e individual. Las propiedades físicas celulares como tamaño (FS) y complejidad interna (SS) permite el reconocimiento de poblaciones celulares considerando características morfológicas que en conjunto con el uso de AcMo conjugados a diferentes fluorocromos nos permiten observar subpoblaciones celulares basados en fenotipos mediante el reconocimiento de proteínas dispuestas en la membrana e intracelularmente. (Véase Figura $\mathrm{N}^{\circ} 1$ )

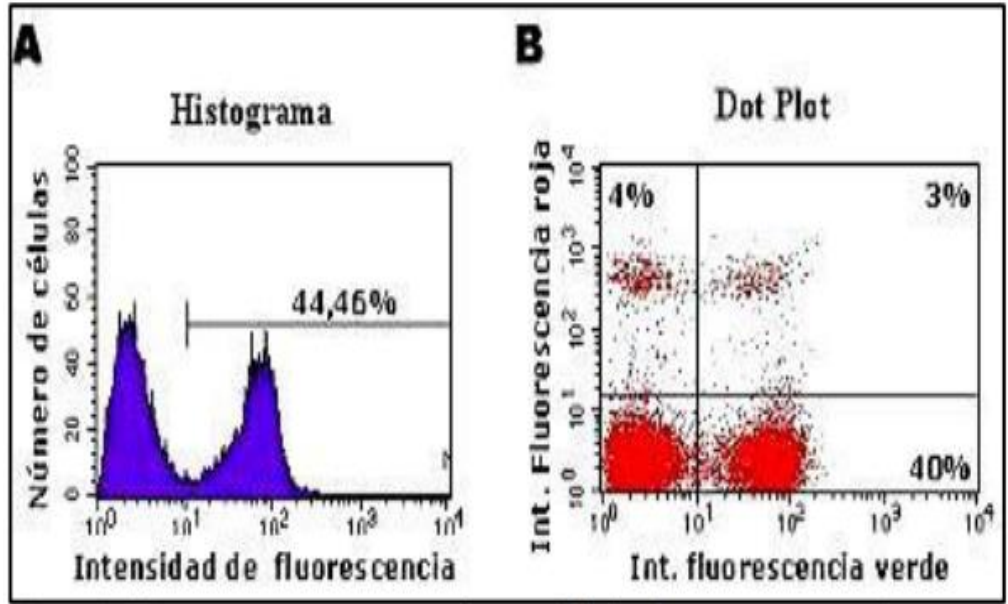

\section{A) Histograma que representa la intensidad de fluorescencia que es directamente proporcional a la cantidad de antígeno presente. (B) Diagrama de puntos (DotPlot), representación simultanea de dos parámetros.}

Componentes del citómetro de flujo

Los componentes básicos de un citómetro de flujo son el sistema hidráulico, el sistema óptico, sistema detector de señales, y el sistema informático: 


\section{Citometría de flujo en el diagnóstico de inmunopatía}

Vol. 3, núm. 1., (2019)

Adrian David Campoverde Cárdenas; Susy Arelis López Laaz; Wilson Pascual Correa Quinto; Jorge Daniel Cárdenas Rodríguez

- Sistema hidráulico: Tiene la responsabilidad del movimiento de la muestra a través de la cámara de flujo desde su aspiración hasta su descarte como desecho. Mediante un enfoque hidrodinámico se crea una suspensión de células simples dentro una vaina liquida. Las células pasan a través del haz láser en fila única a una velocidad aproximada de 500 a 4000 células por segundo.

- Sistema óptico: Consiste de una fuente de excitación y un sistema para generar y coleccionar las señales luminosas. El sistema de excitación consiste en un láser (láser de ion de argón con luz monocromática de $488 \mathrm{~nm}$ ), lentes y prismas para dirigir el rayo. Las características importantes de la luz láser son su alta radiación, estabilidad y pureza espectral.

- El sistema de colección consiste en espejos ópticos y filtros para encaminar determinadas longitudes de onda hacia detectores ópticos determinados. - Sistema electro-informático: Convierte las señales de dispersión de luz y fluorescencia en señales eléctricas y las procesa para su análisis.

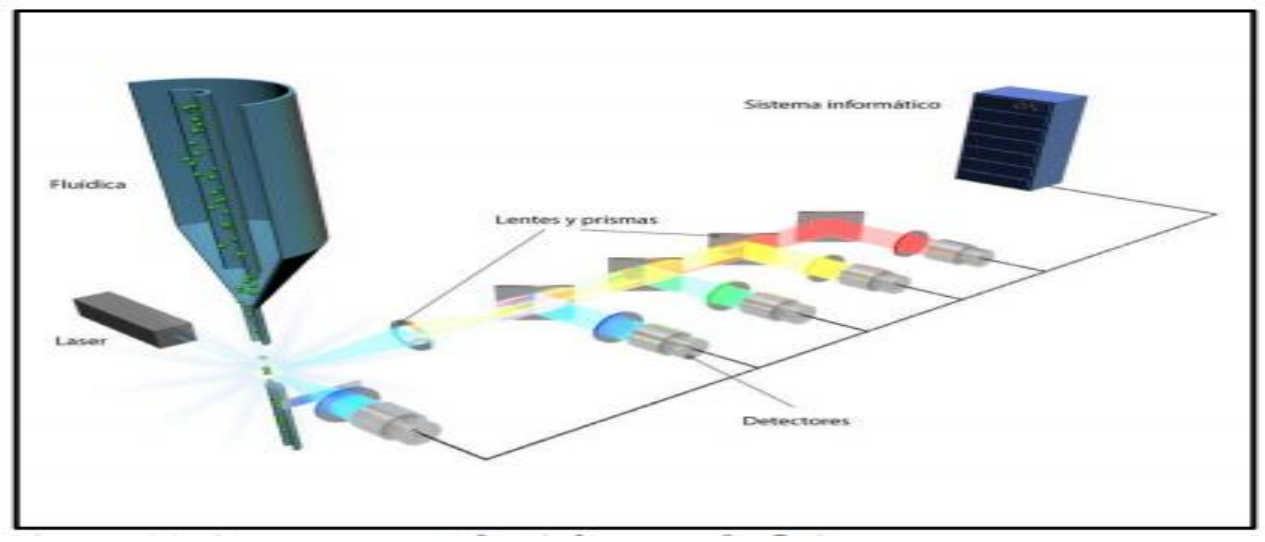




\section{Citometría de flujo en el diagnóstico de inmunopatía}

Vol. 3, núm. 1., (2019)

Adrian David Campoverde Cárdenas; Susy Arelis López Laaz; Wilson Pascual Correa Quinto; Jorge Daniel Cárdenas Rodríguez

\section{Componentes de Citómetro de Flujo}

Aplicaciones más utilizadas de la Citometría de Flujo en el diagnóstico clínico como son la determinación de Antígenos Celulares y la cuantificación de Ácidos Nucleicos

1.- Detección y cuantificación de antígenos La producción de un número cada vez mayor de anticuerpos monoclonales dirigidos frente a epitopos de antígenos presentes en células humanas ha impul116 sado enormemente la utilización de la citometría de flujo para la identificación y clasificación de células tanto normales como patológicas. Su empleo se basa en la identificación de antígenos mediante anticuerpos monoclonales marcados con fluorocromos. El empleo de fluorocromos que siendo excitados en longitudes de onda similares emite en diferente zona del espectro luminoso, amplía a su vez el campo de aplicaciones de la citometría de flujo al poder combinar el uso en una misma muestra de diferentes anticuerpos monoclonales. El isotiocianato de fluoresceína, la ficoeritrina y el PerCP son en la actualidad los fluorocromos más empleados en el marcaje de anticuerpos monoclonales. (Chen, 2013)

Todos ellos se excitan a 488nm (luz azul) si bien emiten en la zona del verde, naranja y rojo, respectivamente. La pequeña superposición de los espectros de emisión se corrige mediante una compensación electrónica realizada en el citómetro, permitiendo su utilización simultánea. No obstante, mediante esta metodología se han logrado importantes avances en el diagnóstico clínico, destacando la aportación de la citometría de flujo al estudio de las subpoblaciones linfocitarias y al diagnóstico y clasificación de las leucemias y linfomas. Así mismo, el empleo de esta tecnología ha demostrado ser de gran utilidad en otras áreas como la detección de autoanticuerpos e inmunocomplejos, el diagnóstico de trombocitopatías adquiridas, la realización 


\section{Citometría de flujo en el diagnóstico de inmunopatía}

Vol. 3, núm. 1., (2019)

Adrian David Campoverde Cárdenas; Susy Arelis López Laaz; Wilson Pascual Correa Quinto; Jorge Daniel Cárdenas Rodríguez

del test cruzado linfocitario, el diagnóstico diferencial de neoplasias epiteliales, la detección tanto de oncoproteínas como de receptores celulares para factores de crecimiento y hormonas y la identificación de subpoblaciones celulares que representan únicamente una pequeña proporción de la celularidad global.(Chen, 2013)

l-a.- Subpoblaciones linfocitarias: La monitorización de las subpoblaciones linfocitarias tiene hoy especial relevancia en el síndrome de inmunodeficiencia adquirida (SIDA) debido por un lado a la expansión experimentada en la última década por el virus de la inmunodeficiencia humana y por otra parte a que la cuantificación en sagre periférica del número de células $\mathrm{T}$ CD4+ y de los linfocitos CD8+/CD38+ posee en estos pacientes gran valor diagnóstico y pronóstico. Estos factores han contribuído de forma decisiva a que la determinación de las poblaciones linfocitarias represente una técnica de rutina en muchos laboratorios. Así mismo, la utilidad de esta determinación ha quedado ampliamente demostrada en otras áreas como en el diagnóstico y clasificación de las inmunodeficiencias primarias, en la monitorización de enfermedades autoinmunes como la esclerosis múltiple. (Chen, 2013)

Por su parte, el lupus eritematoso sistémico, la artritis reumatoide, la artritis psoriásica o la enfermedad de Graves-Basedow, en el seguimiento de la recuperación inmune tras transplante de médula ósea, en la identificación precoz del rechazo en individuos que han recibido un transplantealogénico, o en la monitorización terapeútica de los pacientes con anemia aplásica.Finalmente la utilidad de la determinación de las subpoblaciones linfocitarias en líquidos de lavados broncoalveolares con fines diagnósticos ha sido sugerida en la sarcoidosis donde se observa un predominio claro de células CD4+/CD29+/CD45RA- y en la neumonitis por 


\section{Citometría de flujo en el diagnóstico de inmunopatía}

Vol. 3, núm. 1., (2019)

Adrian David Campoverde Cárdenas; Susy Arelis López Laaz; Wilson Pascual Correa Quinto; Jorge Daniel Cárdenas Rodríguez

hipersensibilidad, al existir en estos pacientes un notable incremento de los linfocitos T activados y de las células T CD8+/CD57+.(Giorgi, 2015)

l-b.- Inmunofenotipaje de leucemias y linfomas: Desde hace tiempo se conoce la utilidad del inmunofenotipo en la clasificación de las leucemias linfoblásticas agudas así como de las crisis blásticas de los síndromes mieloproliferativos y mielodisplásicos con sus correspondientes implicaciones terapéuticas y pronósticas. En el caso de las leucemias mieloblásticas agudas, la importancia del inmunofenotipaje resulta evidente en el diagnóstico de las leucemias megacarioblásticas y de la variante micro granular de la leucemia promielocítica que morfológicamente pueden plantear problemas diagnósticos y en la clasificación de estas leucemias a través de la defmición de la expresión de antígenos frente a las diferentes líneas mieloides y sus estadios madurativos. Así mismo, la reactividad para algunos antígenos -CD34, CDllb, CD7- se ha relacionado con el pronóstico de la enfermedad. (Giorgi, 2015)

El empleo de la citometría de flujo en el estudio de los síndromes linfoproliferativos crónicos posee especial relevancia al permitir un diagnóstico diferencial rápido entre una linfocito si s reactiva y un proceso monoclonal y en este caso contribuir a su filiación diagnóstica. En este sentido, el empleo del triple marcaje CD 19/kappa/lambda es de gran utilidad en la detección de monoclonalidad B en sangre periférica, médula ósea, ganglio o bazo. Así mismo, la utilización de diferentes combinaciones de anticuerpos monoclonales permite diferenciar dentro de las linfocitosis crónicas de linfocitos grandes granulares los procesos de origen T CD3+/TCR+ de los de células NK CD3-ITCR-. (Giorgi, 2015) 


\section{Citometría de flujo en el diagnóstico de inmunopatía}

Vol. 3, núm. 1., (2019)

Adrian David Campoverde Cárdenas; Susy Arelis López Laaz; Wilson Pascual Correa Quinto; Jorge Daniel Cárdenas Rodríguez

l-c.- Otras aplicaciones de la determinación de antígenos: La utilización de las técnicas de inmunofluorescencia para la detección de inmunoglobulinas humanas mediante citometría de flujo ha abierto las puertas a la utilización de esta tecnología como método de rutina en el diagnóstico de diferentes enfermedades autoinmunes como la púrpura trombopénica idiopática, las anemias hemolíticas y las neutropenias autoinmunes. Por otra parte, su empleo para la realización del test cruzado linfocitario constituye en la actualidad el método de mayor sensibilidad y especificidad para demostrar, previamente a un trasplante, la presencia de anticuerpos preformados dirigidos frente a antígenos HLA de clase 1 y II, antígenos de células del endotelio vascular y del sistema monocítico. (Chen, 2013)

La identificación mediante citometría de flujo de la ausencia de expresión en la membrana plaquetaria de reactividad para las glicoproteínas Ilb/llIa y lb se viene utilizando desde hace algún tiempo para el diagnóstico de trombocitopatías congénitas como la enfermedad de Glanzman y el síndrome de Bernard119 Soulier, respectivamente. En este campo, la posibilidad de cuantificar el número de moléculas de un antígeno en la membrana plaquetaria y por lo tanto de conocer la densidad de expresión de una determinada glicoproteina, está revolucionando el estudio de la patología plaquetaria. Así, a modo de ejemplo, en la actualidad pueden cuantificarse diferentes glicoproteinas -CD62, CD63- y la conformación activada de otras CD4la activado-, cuya expresión se ha relacionado con la activación plaquetaria. (Chen, 2013)

2.- Cuantificación de ácidos nucleicos La cuantificación de ácidos nucleicos mediante el empleo de diferentes compuestos químicos fluorescentes capaces de unirse de forma específica al ADN, al ARN o a ambos representa desde el punto de vista cronológico, una de las primeras aplicaciones de la citometría de flujo. 


\section{Citometría de flujo en el diagnóstico de inmunopatía}

Vol. 3, núm. 1., (2019)

Adrian David Campoverde Cárdenas; Susy Arelis López Laaz; Wilson Pascual Correa Quinto; Jorge Daniel Cárdenas Rodríguez

2-a.- Cuantificación de ADN: En la actualidad existe un gran número de fluorocromos capaces de unirse al ADN celular. De ellos el yoduro de propidio y el bromuro de etidio son los de uso más extendido en citometría de flujo. Ambos se excitan a longitudes de onda de 488 (luz disponible en la mayoría de los citómetros de flujo) y se unen de forma estequeométrica al ADN de doble cadena. Su principal problema radi120 ca en que se unen también al ARN de doble cadena lo que hace aconsejable tratar previamente a las células con ARNasa. Además, para su tinción con estos fluorocromos la membrana de las células necesita haber sido fijada y/o permeabilizada, con lo que su utilización se limita a células que no vayan a ser separadas y cultivadas posteriormente. (Weber, 2016)

En el caso del naranja de acridina no se presentan los problemas anteriores ya que este fluorocromo puede ser utilizado para teñir el ADN en células vivas y la fluorescencia debida a su unión a ácidos nucleicos de cadena simple -gran parte del ARN celular- se detecta en distinta zona del espectro luminoso -naranja- en relación con la debida a la unión con la mayoría del ADN celular verde o sin embargo, este fluorocromo presenta el inconveniente de unirse al material plástico del citómetro de flujo creando problemas en mediciones posteriores.Especial referencia merecen también el empleo entre otros del Hoechst y de la cromomicina-A3 que de forma específica se unen a pares de bases adenina/timina o citosina/guanina, respectivamente y de los análogos de la timidina como la bromodeoxiuridina o la yododeoxiuridina, incorporados de forma específica por las células que están sintetizando ADN. (Weber, 2016)

En líneas generales, la cuantificación de ADN proporciona dos tipos de información biológica distintos: por un lado nos orienta sobre la existencia o no de anomalías clonales de ADN -aneuploidias de ADN- y, por otra parte nos permite conocer la distribución de una 


\section{Citometría de flujo en el diagnóstico de inmunopatía}

Vol. 3, núm. 1., (2019)

Adrian David Campoverde Cárdenas; Susy Arelis López Laaz; Wilson Pascual Correa Quinto; Jorge Daniel Cárdenas Rodríguez

población a 10 largo de las distintas fases del ciclo celular. La gran cantidad de estudios clínicos realizados hasta la fecha han puesto de manifiesto que además de proporcionar una información de gran valor sobre la biología de células normales y patológicas, la cuantificación del ADN celular mediante citometría de flujo posee gran impacto clínico en el área de la patología tumoral al contribuir al diagnóstico y a la valoración pronóstica de estos pacientes.(Weber, 2016)

2-b.- Cuantificación de ARN: La cuantificación de ARN mediante citometría de flujo empleando diferentes fluorocromos como el naranja de tiazol, la tioflavina $\mathrm{T}$ o la pironina $\mathrm{Y}$ tiene especial relevancia en el diagnóstico clínico por su utilización como técnica de rutina para el recuento de reticulocitos, con el fin de conocer el equilibrio entre producción y destrucción de eritrocitos y en líneas generales la producción celular de la médula ósea. En relación con las técnicas clásicas, la citometría de flujo ofrece no sólo la posibilidad de realizar un recuento de reticulocitos de gran precisión (contaje de 10.000 células en pocos segundos) sino que además proporciona una información sobre el contenido relativo de ARN de cada reticulocito detectado lo que refleja en gran medida su estadío madurativo. (Leniutt, 2015)

Pese a que esta sea la aplicación más extendida de la cuantificación de ARN en el diagnóstico clínico hay que recordar que la cuantificación simultánea de ADN y ARN mediante citometría de flujo proporciona también una información de gran utilidad para el conocimiento de la actividad metabólica celular que en ocasiones posee importante repercusión clínica. Así, estudios recientes han demostrado la utilidad de esta determinación en el estudio in vitro del efecto sinérgico de diferentes drogas antiblásticas y factores de crecimiento celular.Leniutt, 2015) 


\section{Citometría de flujo en el diagnóstico de inmunopatía}

Vol. 3, núm. 1., (2019)

Adrian David Campoverde Cárdenas; Susy Arelis López Laaz; Wilson Pascual Correa Quinto; Jorge Daniel Cárdenas Rodríguez

\section{3.- Otras aplicaciones de la citometría de flujo: En la actualidad las aplicaciones} experimentales de la citometría de flujo son muy diversas. De ellas hay algunas que presentan una clara utilidad clínica destacando el cariotipo de flujo, la separación de cromosomas y los estudios de hibridación in situ para secuencias específicas de ADN y/o ARN. 122 El cariotipo de flujo representa un complemento y a la vez una alternativa a los métodos clásicos de estudio de los cromosomas en metafase. Para ello los cromosomas son aislados a partir de preparaciones de metafases y teñidos con un fluorocromo -análisis unidimensional- o dos -estudio bidimensional-, empleándose habitualmente el yoduro de propidio o el bromuro de etidio y la combinación del Hoechst 33258 y la cromomicina-A3, respectivamente.Leniutt, 2015).

Finalmente, los cromosomas en suspensión son analizados al pasar de uno en uno por delante de la fuente de luz del citómetro. En relación con otras técnicas, la citometría de flujo ofrece ciertas ventajas que hacen de ella una alternativa válida para la detección de anomalías cromosómicas. En este sentido, proporciona una elevada precisión analítica -en pocos minutos pueden analizarse más de 100.000 cromosomas- y una alta capacidad de resolución -pennite distinguir diferencias en el contenido de ADN de un cromosoma superiores a 2 megabases-o Por ello, además de su potencial empleo en la identificación de anomalías cromosómicas compensadas de gran valor diagnóstico como la traslocación 9/22 de la leucemia mieloide crónica, su utilidad es manifiesta en el estudio de los polimorfismos individuales y de los "microcromosomas" humanos. (Leniutt, 2015). 


\section{Citometría de flujo en el diagnóstico de inmunopatía}

Vol. 3, núm. 1., (2019)

Adrian David Campoverde Cárdenas; Susy Arelis López Laaz; Wilson Pascual Correa Quinto; Jorge Daniel Cárdenas Rodríguez

\section{Conclusiones.}

La aplicación de estudios realizados a través de la técnica de citometría de flujo representan en la actualidad un apoyó clínico de gran valor, a través de los mismos pueden desarrollarse estudios de los marcadores de las leucemias linfoides, los cuales han mejorado enormemente la precisión del diagnóstico, ya que proveen información específica que toma en cuenta el linaje y el estado de maduración de las células malignas, aumentando el conocimiento del médico tratante.

De allí, que alcanzar el conocimiento de las características inmunológicas de las células leucémicas ha sido esencial para la generación de la respuesta fenotipo específica en el contexto de la terapia moderna y los desórdenes linfoproliferativos crónicos, aunque la información derivada del inmunofenotipo es esencial para definir el tipo de población linfoide y es útil para distinguir entre varias poblaciones, la clínica, citología y las características histopatológicas de las enfermedad también son importantes para establecer un diagnóstico más preciso. Dicho de otra forma, el campo de aplicación de este tipo de instrumentos crece rápidamente día a día ya que son numerosas las áreas de la biología y medicina que se benefician de su uso. Entre otras aplicaciones, se encuentra la tipificación de las leucemias y los linfomas que permite alcanzar un diagnóstico preciso, permitiendo un tratamiento y seguimiento más adecuado y eficaz.

Otra aplicación muy importante es el estudio de las poblaciones linfocitarias afectadas por el virus de la inmunodeficiencia humana (VIH) causante del Síndrome de Inmunodeficiencia Adquirida (SIDA) que permite conocer en los individuos afectados su capacidad de respuesta inmunitaria, de capital importancia para el seguimiento de la evolución de la infección y el 


\section{Citometría de flujo en el diagnóstico de inmunopatía}

Vol. 3, núm. 1., (2019)

Adrian David Campoverde Cárdenas; Susy Arelis López Laaz; Wilson Pascual Correa Quinto; Jorge Daniel Cárdenas Rodríguez

tratamiento adecuado para el paciente. En definitiva, es importante entender que la obtención de suspensiones cromosómicas de elevada pureza mediante citometría de flujo ha permitido formidables avances en el conocimiento del genoma humano y en la obtención de sondas moleculares aplicables a diagnóstico citogenético de diversas patologías.

\section{Bibliografía.}

Barrera, S. (2015). Onset Atrial Fibrillation in Hypertensive. Ventricular Hypertrophy. Circ Arrhythm, 47.

Bolívar, L. (2010). Metodología de la Investigación. Caracas: Panapo.

Bolívar, S. (2015). Paradigmas de la Investigación Científica. Pereira, Colombia: Nuevas Fuentes.

Carbajo, E. (2015). Circadian variation in the distribution of analyzed by flow cytometry. J. Endocrinol. México: $2^{a}$ ed. Ed.McGraw Hill-Interamericana.

Cardiología, S. A. (2007). Pathophysiology, and Treatment of Atrial. Cardiol Rev, 181.

Carreño, J. (2015). Complicaciones médicas de La Fibrilación Atrial. Revista de Salud de la Escuela de Medicina de la Universidad del Zulia LUZ, Maracaibo - Venezuela, 13.

Castro, E. (2012). Paradigma y Diseño de la Investigación Científica. España: Las Luces.

Catro, C. (2016). Diseño y enfoque de la Investigación Científica. Madrid: Capital.

Chen, J. (2013). Linfopoyesis temprana en médula ósea adulta. Revista Medica Panamericana, $23,24$.

Colmenárez, M. (2014). Propiedades físicas y celulares de la Célula. Revista Salud de la Universidad de Los Andes MÉRIDA VENEZUELA., 23.

Dávila, P. (2015). Metodología de la Investigación Científica. Caracas : Panapo.

Dávila, R. (2013). El diseño de campo en la Investigación Cualitativa. Colombia: Pereira.

Elizari, Y. (2005). Fibrilación auricular: estamos ante una Epidemia? Rev Española de Cardiología, 76.

Giorgi, V. (2015). Lymphocyte subset alterations and. Clin. ImmunoL, 12. 


\section{Citometría de flujo en el diagnóstico de inmunopatía}

Vol. 3, núm. 1., (2019)

Adrian David Campoverde Cárdenas; Susy Arelis López Laaz; Wilson Pascual Correa Quinto; Jorge Daniel Cárdenas Rodríguez

Guerra, H. (2012). Epidemiology of Atrial Fibrillation i. Rev Española Caiolog, 45.

Jara, L. (2015). Perfil Clínico de la Atrial Fibrillation. Circ Arrhythm, 67.

Leniutt, K. (2015). Aplicaciones de la Citometría de Flujo. Review of the Epidemiology., 33.

Lera y Suatt. (2015). Status of the Epidemiology of Atrial. Med Clin North, 17 - 40.

Leroutt, E. (2014). Epidemiology of Atrial Fibrillation in Spain in the Past 20 Years. Rev Esp Cardiol 2014, 23.

Liendo, A. (2015). Fibrilación auricular en pacientes con insuficiencia cardíaca. Rev Fed Arg Cardiol, 32.

Lintz, K. (s.f.). La C ITOMETRÍA EN EL DIAGNÓSTICO DE INMUNOPATÍA.

Lucas, D. (2010). The fibrosis and atrial fibrillationIs the. Barcelona - España: Luces.

Mayani, H. y. (2015). Hematología: la sangre y sus enfermedades. USA: $2^{\mathrm{a}}$ ed. USA: Cambridge University Press;.

Natt. (2012). Estudio de la etiología y factores de riesgo asociados en una muestra de 300 pacientes,. Rev Esp Cardiol , 14.

OMS, O. M. (2013). Causas de la Fibrilación Atrial. Revista Médica La Habana, 23.

Organización Mundial de la Salud OMS. (2014). Estudios sobre la Fibrilación Atrial. Revista Salud de la Universidad de Los Andes MÉRIDA VENEZUELA, 55.

Organización Mundial de la Salud, O. (2016). La Fibrilación Atrial un Evento cardiovascular de efectos negativos al Sistema Sanitario. Revista La Habana, 7.

Paredes, R. (2015). ILa Citometría en el diagnóstico de Flujo. Revista TODO SALUD de la Escuela de Medicina de la Universidad del Zulia, 15.

Peñate. (2013). Ethnicity and stroke risk in Fibrilación Trial,. Rev Fed Arg Cardiol, 35.

Quezada, M. (2014). The Global Burden of Atrial Fibrillation. Review of the Epidemiology of Atrial, 76.

Riera, V. (2014). Etiología de la Fibrilación Atrial. Revista Médica de la Universidad de la Salud de México, 12.

Weber, G. (2016). Cuantificación del ADN Celular. Revista Médica de la Universidad de la Saluden México, 24. 


\section{Citometría de flujo en el diagnóstico de inmunopatía}

Vol. 3, núm. 1., (2019)

Adrian David Campoverde Cárdenas; Susy Arelis López Laaz; Wilson Pascual Correa Quinto; Jorge Daniel Cárdenas Rodríguez

Wilson K. (2013). monitoring of candidate leukemic stem cells defined by the immunophenotype. USA: Haematologica 2010.

Zava, C. (2013). Risk Factors for Fibrilación Atrial. Thoracic Surgery. Arch Bronconeumol, 54. 